\title{
BMJ Open Community-deliverable exercise and anxiety in adults with arthritis and other rheumatic diseases: a protocol for a systematic review and meta-analysis of randomised controlled trials
}

\author{
George A Kelley, ${ }^{1}$ Kristi S Kelley, ${ }^{2}$ Leigh F Callahan ${ }^{3,4}$
}

To cite: Kelley GA, Kelley KS, Callahan LF. Communitydeliverable exercise and anxiety in adults with arthritis and other rheumatic diseases: a protocol for a systematic review and metaanalysis of randomised controlled trials. BMJ Open 2017;7:e014957.

doi:10.1136/bmjopen-2016014957

- Prepublication history and additional material is available. To view please visit the journal (http://dx.doi.org/ 10.1136/bmjopen-2016014957).

Received 29 0ctober 2016 Revised 26 January 2017 Accepted 9 February 2017

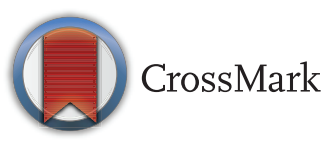

For numbered affiliations see end of article.

Correspondence to Professor George A Kelley; gkelley@hsc.wvu.edu

\section{ABSTRACT}

Introduction: While anxiety is a major public health problem in adults with arthritis and other rheumatic diseases (AORD), the effects of exercise on anxiety in adults are not well established despite numerous studies on this topic. The purpose of this study is to conduct a systematic review with an aggregate data meta-analysis to determine the effects of communitydeliverable exercise interventions (aerobic, strength training or both) on anxiety in adults with AORD.

Methods and analysis: Randomised controlled exercise intervention trials $\geq 4$ weeks and published in any language up to 31 December 2016 will be included. Studies will be retrieved by searching 8 electronic databases, cross-referencing and expert review. Dual selection and abstraction of data will occur. The primary outcome will be changes in anxiety. Risk of bias will be assessed using the Cochrane risk of bias assessment instrument while confidence in the cumulative evidence will be assessed using the Grading of Recommendations Assessment, Development and Evaluation (GRADE) instrument. Standardised effect sizes for anxiety will be calculated from each study and then pooled using the inverse variance heterogeneity (IVhet) model. Meta-regression based on the IVhet model will be used to examine the relationship between changes in anxiety and selected covariates.

Dissemination: The results of this study will be presented at a professional conference and published in a peer-reviewed journal.

Trial registration number: CRD42016048728.

\section{INTRODUCTION}

\section{Rationale}

Arthritis and other rheumatic diseases (AORD) are major public health problems in the USA. Based on combined 2010-2012 data from the National Health Interview Survey, the annual prevalence of doctor-diagnosed arthritis in the civilian, non-institutionalised

\section{Strengths and limitations of this study}

- To the best of our knowledge, this is the first systematic review with meta-analysis to use the inverse variance heterogeneity model to examine the effects of exercise on anxiety as a primary outcome in adults with arthritis and other rheumatic diseases (AORD).

- The results of this systematic review with meta-analysis will be useful to practitioners for making informed decisions about the role of exercise in the treatment of anxiety in adults with AORD as well as provide researchers with direction for the conduct and reporting of future research on this topic.

- Common to most meta-analyses, the results may yield significant heterogeneity which cannot be explained.

US population aged 18 years or older was $22.7 \%$ (52.5 million), with prevalence higher among women $(23.9 \%)$ than men $(18.6 \%) .{ }^{1}$ By the year 2030, it is estimated that the prevalence of doctor-diagnosed arthritis in adults aged 18 years and older will increase to $\sim 67$ million. ${ }^{2}$ Compared with the combined 2010 2012 data, ${ }^{1}$ this represents an increase of 14.5 million adults. Not surprisingly, the financial costs associated with AORD in the USA are high. ${ }^{1}$ In 2003, the total costs attributable to AORD were estimated to be $\sim \$ 128$ billion, $\$ 80.8$ billion in direct costs (medical expenditures) and $\$ 47.0$ billion in indirect costs (lost earnings). ${ }^{3}$ This represents an increase of $24 \%$ between 1997 and $2003 .^{3}$

Elevated and sustained levels of anxiety can result in a number of deleterious consequences. These include, but are not limited to: (1) an increased risk for coronary heart disease as a result of heightened arousal leading to an increased risk for hypertension and a proinflammatory state,,$^{4-6}$ (2) an 
increased risk for cardiac death, ${ }^{5}(3)$ a poorer quality of life, ${ }^{7}$ and (4) a poorer psychosocial functioning. ${ }^{7}$

While it is well recognised that depression is a common comorbidity among adults with AORD, recent research suggests that the prevalence of anxiety among US adults with arthritis is approximately twice as high as depression. ${ }^{8}$ Using data from the Arthritis Conditions Health Effects Survey, the prevalence of anxiety and depression among US adults with doctor-diagnosed arthritis was estimated to be almost twice as high for anxiety $(30.5 \%)$ versus depression $(17.5 \%)$, with US population estimates of 11.5 million for anxiety and 6.6 million for depression. ${ }^{8}$ Given the prevalence of anxiety, it was recommended that healthcare providers screen people with arthritis for anxiety. ${ }^{8}$

Exercise is an intervention that is generally safe and appropriate for most persons with various types of AORD. ${ }^{9}{ }^{10}$ Recent meta-analytic work has shown that community-deliverable exercise interventions reduce depressive symptoms in adults, with an estimated 3.1 million inactive US adults with AORD improving their depressive symptoms if they began and maintained a regular exercise programme. ${ }^{11}$ However, the effects of community-deliverable exercise on anxiety as a primary outcome are not known given a plethora of conflicting randomised controlled trials on this topic as well as a lack of studies that assess both depression and anxiety within the same study (only 44.8\%). ${ }^{11}$ Most importantly, a recent systematic review of previous meta-analyses, not to be confused with an original systematic review with meta-analyses, found that no meta-analysis of randomised controlled trials has examined the effects of communitydeliverable exercise on anxiety as a primary outcome in adults with AORD. ${ }^{12}$ Clearly, it is critically important to develop a better understanding of the overall magnitude of effect, as well as factors associated with exercise for improving anxiety in adults with AORD.

\section{Objective}

The primary objective of this study is to conduct a systematic review with an aggregate data meta-analysis of randomised controlled trials to determine the effects of community-deliverable exercise interventions on anxiety in adults with AORD.

\section{METHODS}

\section{Overview}

This study will follow the guidelines from the Preferred Reporting Items for Systematic Reviews and Meta-Analysis (PRISMA) statement for meta-analyses of healthcare interventions ${ }^{13}$ and the current protocol report follows the Preferred Reporting items for Systematic Review and Meta-Analysis Protocols (PRISMA-P). ${ }^{14}{ }^{15}$ This protocol is registered in PROSPERO (trial registration number CRD42016048728).

\section{Eligibility criteria}

Studies that meet the following criteria will be included: (1) randomised controlled trials with the unit of assignment at the participant level, (2) community-deliverable exercise-only intervention group (aerobic, strength training or both), (3) interventions $\geq 4$ weeks, (4) comparative control group (non-intervention, wait-list control, usual care, attention control), (5) adults $\geq 18$ years of age with doctor-diagnosed osteoarthritis, rheumatoid arthritis or fibromyalgia, (6) studies (published and unpublished in the form of Master's theses and dissertations) in any language, assuming an English language abstract is available between 1 January 1981 and 31 December 2016, and (7) data for anxiety, as defined by the authors of the original studies. Studies will be limited to those with osteoarthritis, rheumatoid arthritis or fibromyalgia based on our previous research showing a lack of exercise intervention studies for other types of AORD. ${ }^{11}$ Studies will be limited to randomised trials because it is the only way to control for confounders that are not known or measured as well as the observation that non-randomised controlled trials tend to overestimate the effects of healthcare interventions. ${ }^{16}{ }^{17}$ Aerobic and progressive resistance (strength training) exercise will be defined according to section C2 of the 2008 Physical Activity Guidelines Advisory Committee Report. ${ }^{18}$ Specifically, aerobic exercise is defined as any 'exercise that primarily uses the aerobic energyproducing systems, can improve the capacity and efficiency of these systems, and is effective for improving cardiorespiratory endurance', while strength training is defined as 'exercise training primarily designed to increase skeletal muscle strength, power, endurance and mass'. ${ }^{18}$

For this proposed project, community-deliverable exercise interventions will be those that could be performed, or have the potential to be adapted and performed, by persons in a community setting (recreation or senior centres, in the home or neighbourhood, etc.) and meet the implementation guidelines for physical activity interventions recently recommended by the Arthritis Program at the Centers for Disease Control and Prevention: (1) no academic degree required for a leader/implementer but leader training available, if needed, (2) no special facilities beyond a community room (except a warm pool for aquatic exercise), (3) inexpensive equipment, (4) cost to participants $<\$ 50.00$, (5) implementation guide available, (6) supporting structures judged to be adequate to support widespread implementation. ${ }^{19}$ An exercise duration of at least 4 weeks was chosen based on previous research in which statistically significant improvements in anxiety occurred as a result of as little as 4 weeks of exercise training. ${ }^{20}$ There will be no maximum limit on the length of any interventions for the studies included in our proposed project. We will also limit our studies to adults aged 18 years and older because the inclusion of children and adolescents poses additional confounding problems 
congruent with the many developmental changes that occur during this period. In addition, the prevalence of AORD is more common in adults than in children and adolescents. We will restrict our studies to published articles, dissertations and Master's theses and examine for potential small-study effects such as publication bias when limited to published articles in peer-reviewed journals. The year 1981 was chosen as the starting point for eligibility based on a preliminary PubMed search in which it was found that this was the first year that a randomised controlled trial on exercise and arthritis was published. ${ }^{21}$ Studies from both English and non-English language sources will be included with the latter translated into English by the research technician using the freely available web-based Babelfish and Bing translators. For those studies that cannot be translated using Babelfish and Bing, we will use professional translation services to accomplish such translation. Finally, while acknowledging that the mechanisms for changes in anxiety may differ for different types of AORD, for example, fibromyalgia versus osteoarthritis and rheumatoid arthritis, the purpose of the current systematic review with meta-analysis is to determine whether exercise reduces anxiety in adults with AORD, not why it reduces anxiety.

\section{Information sources}

The following eight electronic databases will be searched for potentially eligible studies in any language and published between 1 January 1981 and 31 December 2016: (1) PubMed, (2) SPORTDiscus, (3) Cochrane Central Register of Controlled Clinical Trials (CENTRAL), (4) Cumulative Index to Nursing and Allied Health Literature (CINAHL), (5) PsychInfo, (6) Web of Science, (7) Scopus and (8) ProQuest (Master's theses and dissertations). In addition, cross-referencing from retrieved studies will be conducted. Furthermore, the third author (LFC), an expert on exercise and AORD, will review the reference list for completeness.

\section{Search strategy}

Search strategies will be developed using text words as well as medical subject headings (MeSH) associated with the effects of exercise on anxiety in adults with AORD. Studies in languages other than English will be translated into the English language. The second author (KSK) will conduct all electronic database searches. A copy of a preliminary search strategy using PubMed, including limits, can be found in online supplementary file 1 . This search strategy will be adapted for other database searches.

\section{Study records}

\section{Study selection}

All studies to be screened will be imported into EndNote (V.X8; EndNote (Version X8). New York, NY: Thomson Reuters, 2016) and duplicates removed both electronically and manually by the second author
(KSK). A copy of the database will then be provided to the first author for duplicate screening. The first two authors (GAK and KSK) will select all studies, independent of each other. The full report for each article will be obtained for all titles and abstracts that appear to meet the inclusion criteria or where there is any uncertainty. Multiple reports of the same study will be handled by including the most recently published article as well as drawing from previous reports, assuming similar methods and sample sizes. Neither of the screeners will be blinded to the journal titles or to the study authors or institutions. Reasons for exclusion will be coded as one or more of the following: (1) inappropriate population, (2) inappropriate intervention, (3) inappropriate comparison(s), (4) inappropriate outcome(s), (5) inappropriate study design and (6) other. On completion, the first two authors (GAK and KSK) will meet and review their selections. Discrepancies will be resolved by consensus. If consensus cannot be reached, the third author (LFC) will provide a recommendation. The overall agreement rate prior to correcting discrepant items will be calculated using Cohen's $\kappa$ statistic. ${ }^{22}$ After identifying the final number of studies to be included, the overall precision of the searches will be calculated by dividing the number of studies included by the total number of studies screened after removing duplicates. ${ }^{23}$ The number needed-to-read will then be calculated as the inverse of the precision. ${ }^{23}$ A flow diagram that depicts the search process will be included as well as an online supplementary file that includes a reference list of all studies excluded, including the reason(s) for exclusion. The proposed structure for the flow diagram is shown in figure 1 .

\section{Data abstraction}

Prior to the abstraction of data, a codebook that can hold more than 200 items per study will be developed using Microsoft Excel (Microsoft Excel (Version 2010). Redmond, Washington: Microsoft Corporation, 2010). The codebook will be developed by the first two authors (GAK and KSK) with input from the third author (LFC). The major categories of variables to be coded will include: (1) study characteristics (author, journal, year, etc.), (2) participant characteristics (age, height, body weight, etc.), (3) intervention characteristics (type, length, frequency, intensity, duration, compliance, etc.) and (4) outcome characteristics for anxiety (sample sizes, baseline and postexercise means and SDs, etc.). The first two authors (GAK and KSK) will abstract the data from all studies, independent of each other, using separate codebooks in Microsoft Excel (Microsoft Excel (Version 2010)). On completion of coding, the codebooks will be merged into one primary codebook for review. Both authors will then meet and review all selections for agreement. Discrepancies will be resolved by consensus. If consensus cannot be reached, the third author (LFC) will provide a recommendation. Prior to 


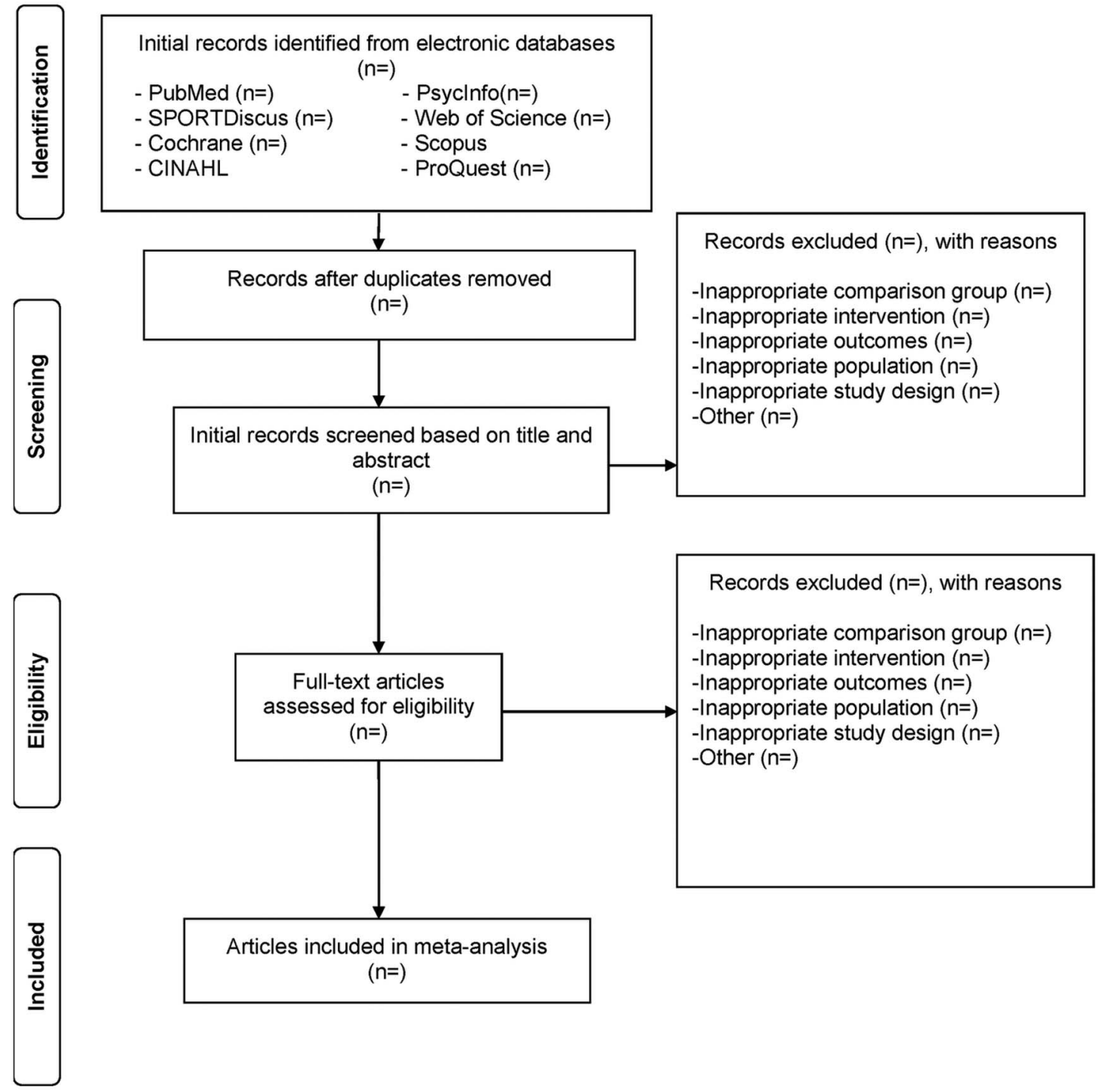

Figure 1 Proposed flow diagram to depict the search process.

correcting disagreements, the overall agreement rate will be calculated using Cohen's $\kappa$ statistic. ${ }^{22}$

\section{Outcomes and prioritisation}

The primary outcome in this study will be changes in anxiety. Secondary outcomes will include changes in physical function, pain, quality of life, depression, body mass index (BMI), aerobic fitness, and upper and lower body strength.

\section{Risk of bias assessment in individual studies}

Risk of bias will be assessed at the study level using the Cochrane risk of bias instrument, ${ }^{24}$ with a focus on the primary outcome of interest, changes in anxiety. Bias will be evaluated for six domains: (1) random sequence generation, (2) allocation concealment, (3) blinding of participants and personnel, (4) blinding of outcome assessment, (5) incomplete outcome data, (6) selective reporting, and (7) whether participants were exercising regularly, as defined by the original study authors, prior to taking part in the study. Each item will be classified as having either a high, low or unclear risk of bias. In addition, a text description of the basis for our judgement in each of the seven domains will be provided. For example, for a study rated as being at an unclear risk of bias for the category of whether or not participants were exercising regularly, we may provide a text description such as insufficient information provided to make a decision'. Assessment for risk of bias will be limited to the primary outcome of interest, that is, changes in anxiety. Since it is virtually impossible to blind participants to group assignment in exercise intervention protocols, all studies will be classified as high risk of bias with respect to the category 'blinding of participants and personnel'. On the basis of previous research, no study will be excluded based on the results of the risk of bias assessment. ${ }^{25}$ The first two authors (GAK and KSK) will conduct all assessments, independent of each other. Both authors will then meet and review all selections for agreement. Discrepancies will be resolved by consensus. 
If consensus cannot be reached, the third author (LFC) will provide a recommendation.

\section{Data synthesis}

\section{Calculation of effect sizes}

The primary outcome for this proposed project will be changes in anxiety, calculated as Hedge's standardised mean difference effect size (ES), adjusted for smallsample bias. ${ }^{26}$ This will be calculated by subtracting the change outcome difference in the exercise group minus the change outcome difference in the control group, and then dividing by the pooled SD of the change outcome for the exercise and control groups. If change score SDs are not available, these will be calculated from reported change outcome or treatment effect $95 \%$ CIs or pre-SD and post-SD values according to procedures developed by Follmann et $a l^{27}$ Secondary outcomes (physical function, pain, quality of life, depression, BMI, aerobic fitness, upper and lower body strength) will be calculated using the same procedures as for our primary outcome. For studies in which outcomes are assessed at multiple time points, we plan on examining differences between baseline values and the final time point closest to cessation of the exercise intervention.

\section{Pooled estimates for changes in outcomes}

ES changes in anxiety and all secondary outcomes will be pooled using the recently developed inverse heterogeneity (IVhet) model. ${ }^{28}$ The IVhet model is a quasilikelihood model that is computed by (1) calculating weights that sum to 1 from each study, (2) pooling effects from all the studies and (3) calculating the variance of the pooled ES. The IVhet model has been shown to be superior to the original random-effects, method-ofmoments model of Dersimonian and Laird, ${ }^{28} 29$ the most common random-effects model used to pool aggregate data meta-analytic results. ${ }^{29}$ Specifically, simulation studies have shown that the IVhet model retains correct coverage probabilities as well as a lower observed variance than the random-effects model, regardless of heterogeneity. ${ }^{28}$ Two-tailed $z-\alpha$ values $<0.05$ as well as non-overlapping $95 \%$ CIs will be considered statistically significant.

Heterogeneity and inconsistency for each pooled outcome will be estimated using the $Q^{30}$ and $\mathrm{I}^{2}$ statistic, ${ }^{31}$ respectively. An $\alpha$ level of $\leq 0.10$ for $Q$ will be considered to represent statistically significant heterogeneity while inconsistency will be categorised as very low $(<25 \%)$, low (25\% to $<50 \%)$, moderate $(50 \%$ to $<75 \%)$ or large $(\geq$ $75 \%) .{ }^{31}$ To improve practical relevance with respect to potential improvements in anxiety and all secondary outcomes, percentile gain in the exercise groups will be calculated using Cohen's $\mathrm{U}_{3}$ index ${ }^{32}$ while the numberneeded-to treat (NNT) will be estimated. ${ }^{33}$

Derived from NNT, gross estimates will also be calculated for the number of adults with AORD in the USA who may benefit from exercise but are not currently meeting exercise recommendations. This will be based on the reciprocal of the NNT multiplied by the number of adults in the USA with doctor-diagnosed arthritis who were not currently meeting exercise guidelines, currently >34.8 million. ${ }^{134}$

Influence analysis will be conducted with each study deleted from the model once in order to examine the effect of each study on our overall results. In addition, cumulative meta-analysis, ranked by year, will be used to examine the accumulation of results over time.

Meta-regression based on the IVhet model will be used to examine the relationship between changes in our primary outcome and selected covariates (table 1)..$^{28}$ Broadly, this will be accomplished by (1) conducting simple meta-regression for statistically significant associations between selected covariates and changes in anxiety, (2) examining for multicollinearity between covariates $(r>0.80)$, and (3) building a multiple meta-regression model (table 1). These models will use a multiplicative versus additive component of residual heterogeneity (Meta XL User Guide (Version 5.3). Queensland, Australia: EpiGear International Pty Ltd, 2016). To achieve matching error variances, robust Huber-Ecker-White-sandwich error variances will be used to account for the underestimated dispersion

Table 1 Covariates to initially examine using simple meta-regression

\begin{tabular}{|c|c|}
\hline Characteristics & Variable \\
\hline Study & $\begin{array}{l}\text { Publication year, impact factor of journal, country study conducted, type of control group, bias (sequence } \\
\text { generation, allocation concealment, blinding of participants and personnel, blinding of outcome assessors, } \\
\text { incomplete outcome data, selective outcome reporting), type of analysis }\end{array}$ \\
\hline Participant & $\begin{array}{l}\text { Age, gender, race/ethnicity, income, education, marital status, type of AORD, years since diagnosis of } \\
\text { AORD }\end{array}$ \\
\hline Exercise & $\begin{array}{l}\text { Type (aerobic, strength, both), length, frequency, intensity, duration, total minutes, total minutes (adjusted } \\
\text { for compliance), mode, compliance, exercise supervision, setting, number of sets, number of repetitions, } \\
\text { rest between sets, number of exercises, type of resistance, equipment used, fidelity (design, training, } \\
\text { delivery, receipt, enactment) }\end{array}$ \\
\hline Outcome & $\begin{array}{l}\text { Baseline values for anxiety, instrumentation, changes in physical function, changes in pain, changes in } \\
\text { quality of life, changes in depression, changes in BMI, changes in aerobic fitness, changes in upper body } \\
\text { strength, changes in lower body strength }\end{array}$ \\
\hline
\end{tabular}


(Meta XL User Guide (Version 5.3). 2016). Such errors are expected to calculate the correct SEs for heterogeneous data that are traditionally heteroscedastic (Meta XL User Guide (Version 5.3). 2016). For this study, continuous variables will be modelled as averages while categorical variables will be modelled as proportions.

Based on the recommendations of Rothman, ${ }^{35}$ no adjustments for multiple testing will be made because of concerns about missing possibly important findings that could be pursued in future randomised controlled trials. ${ }^{35}$ While this could be viewed as a 'fishing expedition', such analyses are important for providing investigators with potential direction for future randomised controlled trials, one of the very reasons for conducting a systematic review with meta-analysis. This approach is especially appropriate for meta-analysis since covariates are not randomly assigned in meta-analysis, and thus such analyses are considered to be observational in nature. ${ }^{36}$ As a result, causal inferences cannot be derived from meta-regression. However, any observed associations can provide direction for future research. Thus, we consider our meta-regression analyses to be exploratory in nature. The former notwithstanding, the investigative team acknowledges that one or more statistically significant associations may be nothing more than chance findings.

\section{Meta-biases}

Small-study effects (publication bias, etc.) for primary and secondary outcomes will be assessed following current guidelines. ${ }^{37}$ This will include qualitative analysis using funnel plots as well as quantitative analysis using Egger's regression-intercept test (one-tailed). Ninety-five per cent CIs that do not include zero (0) will be considered to represent statistically significant small-study effects.

\section{Confidence in cumulative evidence}

Strength of findings for our primary outcome (anxiety) and all secondary outcomes (physical function, pain, quality of life, depression, BMI, aerobic fitness, upper and lower body strength) will be evaluated using the Grading of Recommendations Assessment, Development and Evaluation (GRADE) instrument for meta-analysis. ${ }^{38}$ The quality of evidence will be assessed across the domains of risk of bias, consistency, directness, precision and publication bias. Quality will be judged as high (further research is very unlikely to change our confidence in the estimate of effect), moderate (further research is likely to have an important impact on our confidence in the estimate of effect and may change the estimate), low (further research is very likely to have an important impact on our confidence in the estimate of effect and is likely to change the estimate) or very low (very uncertain about the estimate of effect). This will be accomplished using the same procedures as for study selection and data abstraction. Assessments will be conducted following the same procedures as for the abstraction of data and risk of bias for individual studies.

\section{Software used for data synthesis}

All data will be analysed using Stata (V.14.1; Stata/SE for Windows (Version 14.1). College Station, Texas: Stata Corporation LP, 2015), Microsoft Excel 2010 (Microsoft Excel (Version 2010)), and three add-ins for Excel, Meta XL (V.5.3; Meta XL (Version 5.3). 2016), SSC-Stat (V.2.18; SSC-Stat (Version 2.18). University of Reading, UK: Statistical Services Center, 2007), and EZ-Analyze (V.3.0; EZ Analyze (Version 3.0). Boston, MA: Tim Poynton, 2007).

\section{DISSEMINATION}

The results of this study will be presented at a professional conference and published in a peer-reviewed journal.

\section{Author affiliations}

${ }^{1}$ Department of Biostatistics, School of Public Health, West Virginia University, Morgantown, West Virginia, USA

${ }^{2}$ Department of Biostatistics, School of Public Health, West Virginia University, Morgantown, West Virginia, USA

${ }^{3}$ Departments of Social Medicine and Orthopaedics, University of North Carolina, Chapel Hill, North Carolina, USA

${ }^{4}$ Department of Epidemiology, University of North Carolina, Chapel Hill, North Carolina, USA

Contributors GAK is the guarantor. GAK and KSK drafted the manuscript. GAK and KSK contributed to (1) the development of the data sources to search for relevant literature, including search strategy, (2) selection criteria, (3) data extraction criteria and (4) risk of bias assessment strategy. GAK provided statistical and exercise expertise while LFC provided content expertise on exercise and anxiety in adults with AORD. All three authors read, provided feedback and approved the final manuscript.

Funding This meta-analysis is funded by the National Institutes of Health, National Institute for Arthritis, Musculoskeletal and Skin Diseases, grant number R01AR061346 (GAK, principal investigator). GAK and KSK are also partially supported by the National Institute of General Medical Sciences of the National Institutes of Health, grant number U54GM104942.

Disclaimer The content of this manuscript is solely the responsibility of the authors and does not necessarily represent the official views of the National Institutes of Health.

Competing interests None declared

Provenance and peer review Not commissioned; externally peer reviewed.

Data sharing statement This is a meta-analysis protocol. However, all data from the completed meta-analysis will be available from the corresponding author on request.

Open Access This is an Open Access article distributed in accordance with the Creative Commons Attribution Non Commercial (CC BY-NC 4.0) license, which permits others to distribute, remix, adapt, build upon this work noncommercially, and license their derivative works on different terms, provided the original work is properly cited and the use is non-commercial. See: http:// creativecommons.org/licenses/by-nc/4.0/

\section{REFERENCES}

1. Barbour KE, Helmick CG, Theis KA et al. Prevalence of doctor-diagnosed arthritis and arthritis-attributable activity limitation -United States, 2010-2012. Morb Mortal Wkly Rep 2013;62:869-73.

2. Hootman JM, Helmick CG. Projections of US prevalence of arthritis and associated activity limitations. Arthritis Rheum 2006;54:226-9.

3. Yelin E, Murphy L, Cisternas MG, et al. Medical care expenditures and earnings losses among persons with arthritis and other rheumatic conditions in 2003, and comparisons with 1997. Arthritis Rheum 2007;56:1397-407. 
4. Player MS, Peterson LE. Anxiety disorders, hypertension, and cardiovascular risk: a review. Int J Psychiatry Med 2011;41:365-77.

5. Roest AM, Martens EJ, de Jonge P, et al. Anxiety and risk of incident coronary heart disease: a meta-analysis. J Am Coll Cardiol 2010;56:38-46.

6. Janszky I, Ahnve S, Lundberg I, et al. Early-onset depression, anxiety, and risk of subsequent coronary heart disease: 37-year follow-up of 49,321 young Swedish men. J Am Coll Cardiol 2010;56:31-7.

7. Frost $\mathrm{C}$, Clarke $\mathrm{R}$, Beacon $\mathrm{H}$. Use of hierarchical models for meta-analysis: experience in the metabolic ward studies of diet and blood cholesterol. Stat Med 1999;18:1657-76.

8. Murphy LB, Sacks JJ, Brady TJ, et al. Anxiety and depression among US adults with arthritis: Prevalence and correlates. Arthritis Care Res (Hoboken) 2012;64:968-76.

9. Busch AJ, Schachter CL, Overend TJ, et al. Exercise for fibromyalgia: a systematic review. J Rheumatol 2008;35:1130-44.

10. Hurkmans E, van der Giesen FJ, Vliet Vlieland TP, et al. Dynamic exercise programs (aerobic capacity and/or muscle strength training) in patients with rheumatoid arthritis. Cochrane Database Syst Rev 2009;(4):CD006853.

11. Kelley GA, Kelley KS, Hootman JM. Effects of exercise on depression in adults with arthritis: a systematic review with meta-analysis of randomized controlled trials. Arthritis Res Ther 2015;17:21.

12. Kelley GA, Kelley KS. Effects of exercise on anxiety in adults with arthritis and other rheumatic disease: a systematic review of meta-analyses. J Nov Physiother 2014;4:1-5.

13. Liberati A, Altman DG, Tetzlaff $J$, et al. The PRISMA statement for reporting systematic reviews and meta-analyses of studies that evaluate health care interventions: explanation and elaboration. Ann Intern Med 2009;151:W65-94.

14. Shamseer L, Moher D, Clarke M, et al. Preferred reporting items for systematic review and meta-analysis protocols (PRISMA-P) 2015: elaboration and explanation. BMJ 2015;349:97647.

15. Moher D, Shamseer L, Clarke M, et al. Preferred reporting items for systematic review and meta-analysis protocols (PRISMA-P) 2015 statement. Syst Rev 2015;4:1.

16. Sacks HS, Chalmers TC, Smith H. Randomized versus historical controls for clinical trials. Am J Med 1982;72:233-40.

17. Schulz KF, Chalmers I, Hayes RJ, et al. Empirical evidence of bias: dimensions of methodological quality associated with estimates of treatment effects in controlled trials. JAMA 1995;273:408-12.

18. Physical Activity Guidelines Advisory Committee. Physical Activity Guidelines Advisory Report. Washington DC: U.S Department of Health and Human Services, 2008.

19. Brady TJ, Jernick SL, Hootman JM, et al. Public health interventions for arthritis: expanding the toolbox of evidence-based interventions. $J$ Womens Health (Larchmt) 2009;18:1905-17.
20. Ide MR, Laurindo IMM, Rodrigues AL, et al. Effect of aquatic respiratory exercise-based program in patients with fibromyalgia. Int $J$ Rhuem Dis 2008;11:131-40.

21. Waggoner CD, LeLieuvre RB. A method to increase compliance to exercise regimens in rheumatoid arthritis patients. J Behav Med 1981;4:191-201.

22. Cohen J. Weighted kappa: nominal scale agreement with provision for scaled disagreement or partial credit. Psychol Bull 1968;70:213-20.

23. Lee $\mathrm{E}$, Dobbins M, DeCorby K, et al. An optimal search filter for retrieving systematic reviews and meta-analyses. BMC Med Res Methodol 2012;12:51.

24. Higgins JP, Altman DG, Gotzsche PC, et al. The Cochrane Collaboration's tool for assessing risk of bias in randomised trials. BMJ 2011;343:d5928.

25. Ahn S, Becker BJ. Incorporating quality scores in meta-analysis. $J$ Educ Behav Stat 2011;36:555-85.

26. Hedges LV, Olkin I. Statistical methods for meta-analysis. San Diego, CA: Academic Press, 1985.

27. Follmann D, Elliot $\mathrm{P}$, Suh I, et al. Variance imputation for overviews of clinical trials with continuous response. J Clin Epidemiol 1992;45:769-73.

28. Doi SA, Barendregt JJ, Khan S, et al. Advances in the meta-analysis of heterogeneous clinical trials $\mathrm{I}$ : the inverse variance heterogeneity model. Contemp Clin Trials 2015;45(Pt A):130-8.

29. Dersimonian R, Laird N. Meta-analysis in clinical trials revisited. Contemp Clin Trials 2015;45(Pt A):139-45.

30. Cochran WG. The combination of estimates from different experiments. Biometrics 1954;10:101-29.

31. Higgins JPT, Thompson SG, Deeks JJ, et al. Measuring inconsistency in meta-analyses. BMJ 2003;327:557-60.

32. Cohen J. Statistical power analysis for the behavioral sciences. New York: Academic Press, 1988.

33. Kraemer HC, Kupfer DJ. Size of treatment effects and their importance to clinical research and practice. Biol Psychiatry 2006;59:990-6

34. Shih M, Hootman JM, Kruger J, et al. Physical activity in men and women with arthritis National Health Interview Survey, 2002. Am J Prev Med 2006;30:385-93.

35. Rothman KJ. No adjustments are needed for multiple comparisons. Epidemiology 1990;1:43-6.

36. Littell JH, Corcoran J, Pillai V. Systematic reviews and meta-analysis. New York: Oxford University Press, 2008

37. Sterne JA, Sutton AJ, loannidis JP, et al. Recommendations for examining and interpreting funnel plot asymmetry in meta-analyses of randomised controlled trials. BMJ 2011;343:d4002.

38. Guyatt G, Oxman AD, Akl EA, et al. GRADE guidelines:

1. Introduction-GRADE evidence profiles and summary of findings tables. J Clin Epidemiol 2011;64:383-94. 